\title{
Comparative study of lateral internal sphincterotomy versus local $0.2 \%$ glyceryl trinitrate ointment for the treatment of chronic anal fissure
}

\author{
Md. Ibrahim Siddique, Khandker Manzoor Murshed and M.A. Majid \\ Department of Surgery, Bangabandhu Sheikh Mujib Medical University, Shahbag, Dhaka 1000, Bangladesh.
}

\begin{abstract}
The gold standard surgical treatment of chronic anal fissure is lateral internal sphincterotomy which lowers the resting anal pressure and effectively heals the majority of fissures. Local application of $0.2 \%$ glyceryl trinitrate ointment has been used as an agent for chemical sphincterotomy, causing temporary alleviation of sphincter spasm and allowing the fissure to heal without compromising the anal continence. The aim of the present study was to compare the results of surgical sphincterotomy with that of local $0.2 \%$ glyceryl trinitrate ointment in the treatment of chronic anal fissure. Seventy adult patients between the age of 18 and 50 years with chronic anal fissure were randomized in a prospective trial to receive either surgical sphincterotomy or $0.2 \%$ glyceryl trinitrate ointment locally. Patients were followed up at 2 weeks' interval for 10 weeks. Symptom relief, fissure healing and continence scores were the outcomes assessed. Six patients were excluded for protocol violations. Surgical sphincterotomy was significantly more effective in providing pain relief and was associated with significantly better fissure healing rates at 6 weeks and 10 weeks (both $p<0.001$ ). There were substantial problems with compliance in ointment group related to slow healing and longer time needed for symptomatic relief. Minor incontinence was $6 \%$ in sphincterotomy group and none in ointment group $(\mathrm{p}>0.05)$. Considering early symptomatic relief, rapid fissure healing and better patient compliance surgical sphincterotomy is the treatment of choice for chronic anal fissure.
\end{abstract}

\section{Introduction}

Anal fissure is an elongated ulcer in the long axis of lower anal canal causing significant morbidity due to sharp severe anal pain, especially during defecation. Young adults are the usual sufferers. Though, the exact aetiology of primary anal fissure is still unknown, high resting anal pressure caused by increased internal sphincter tone appears to be the underlying pathological factor ${ }^{1}$. There is a vicious cycle beginning from a tear in the anoderm from forceful dilatation of the anal canal during defecation exposing the underlying internal sphincter muscle that eventually goes into spasm and fails to relax during next bowel movement. Further tearing results in persistent muscle spasm leading to relative ischemia of the anoderm causing persistence of symptoms and impairment of healing. Ninety percent of anal fissures heal with stool softeners, bulking agents and sitz baths ${ }^{2}$. A fissure is defined as chronic when it fails to heal within 6 weeks from an acute attack ${ }^{3}$. Reduction in the internal anal sphincter tone increases anodermal blood flow leading to symptomatic relief and healing of chronic anal fissure ${ }^{4}$. Lateral internal sphincterotomy is successful in reducing the sphincteric tone in $95 \%$ of cases $^{1}$. However, a significant number of patients are reported to develop fecal incontinence following this procedure $^{5}$. Over the past few years, topical application of different pharmacological agents like glyceryl trinitrate, diltiazem, nifedipine are being used to reduce the resting anal tone with eventual healing of chronic anal fissure in a significant number of patients ${ }^{3}$. These pharmacological agents produce reversible chemical sphincterotomy without compromising the anal continence. Glyceryl trinitrate releases nitric oxide that mediates the relaxation of internal sphincter. Local application of $0.2 \%$ glyceryl trinitrate ointment to the distal anal canal is reported to heal chronic anal fissure in a good number of patients ${ }^{3,4}$.

A significant number of patients experience temporary headache and this side effect sometimes reduces compliance. Patients with a long history or who have a sentinel piles are less likely to heal with glyceryl trinitrate ointment ${ }^{6}$. Following surgical 
sphincterotomy healing of fissures occurs in $98 \%$ to $100 \%$ of cases 7,8 . Varying degrees of incontinence ranging from $5 \%$ to $30 \%$ were reported in different studies following surgical sphincterotomy ${ }^{8-10}$.

The main objective of this study was to compare the effectiveness and morbidity of surgical versus chemical sphincterotomy by $0.2 \%$ glyceryl trinitrate ointment in the treatment of chronic anal fissure.

\section{Patients and Methods}

This randomized clinical trial was carried out at the Department of Surgery of Bangabandhu Sheikh Mujib Medical University (BSMMU). Seventy adult patients both male and female between the age of 18 and 50 years with chronic anal fissure from February 2006 through to February 2007 were included in the study. Informed written consent was obtained from the patients after full explanation of the details of the disease process, options of treatment, ultimate outcome, possible side effects and complications and chances of recurrences in either procedure. They were informed of their right to withdraw from the trial at any stage. The protocol was approved by the Institutional Ethics Committee of BSMMU. The protocol was considered violated if the patient failed to come for a regular follow-up or had withdrawn from the study. All patients for whom the protocol was deemed violated were excluded from the final analysis. Patients with primary fissure lasting for more than 6 weeks were included in the study. Those with secondary fissures, fissures complicated with fistula and anal stenosis and patients with previous anorectal surgery were excluded from the study. Patients were randomized into two groups of 35 patients in each group. One group underwent lateral internal sphincterotomy and the other group was prescribed $0.2 \%$ glyceryl trinitrate ointment to apply locally to the distal anal canal twice daily for 8 weeks. In surgical group, the patients underwent lateral internal sphincterotomy under spinal anesthesia after complete pre-operative evaluation. Sphincterotomy was carried out by the open method where a 1-2 cm circumferential incision was made at the anal verge at 5 O'clock position over the free edge of the internal sphincter. Blunt scissor dissection was carried out to open the plane inside and outside the internal sphincter to free it. The free lower edge of the internal sphincter was then grasped, drawn into the wound and a length of sphincter equal to the length of fissure below the level of dentate line was divided either by scissor or by diathermy. Associated sentinel skin tag at the outer end of the fissure and sometimes a fibroepithelial polyp at the inner end were also excised if present. The wounds were left open. Patients of both groups were advised to have sitz baths and to take high fiber diet. They were followed up at 2 weeks' interval for 10 weeks and pain relief, fissure healing, compliance and continence scores were evaluated during each follow-up. Pain relief was defined as complete absence of pain. Healing of fissure was accepted when there was no visual lesion in anoderm. A patient was defined fully continent when there was no soiling of perianal region or undergarments under normal circumstances or during stress. Incontinence to flatus or mucus only while on stress was designated as minor incontinence.

Statistical analysis: Data were compiled and statistical analysis was done with chi-square test $\left(\chi^{2}\right.$ test). All $\mathrm{p}$ values were obtained using standard statistical table.

\section{Results}

Six patients were excluded from the study for protocol violations ( 2 in the surgical group and 4 in the ointment group), leaving 64 patients for data analysis, 33 in the surgical sphincterotomy group and 31 in $0.2 \%$ glyceryl trinitrate ointment group. They were followed up at 2 weeks' interval for 10 weeks. In surgical group (Figure 1) symptomatic relief occurred in $22(66.67 \%)$ patients as compared to $4(12.90 \%)$ patients in $0.2 \%$ glyceryl trinitrate ointment group at the end of $2^{\text {nd }}$ week. No healing of fissure was observed in either group at the first visit. After 6 weeks, symptomatic relief was achieved in all $33(100 \%)$ patients in surgical group and $14(45.27 \%)$ patients in ointment group $(\mathrm{p}<0.001)$. Overall healing was in $28(84.85 \%)$ patients in surgical group and in 11 (35.48\%) patients in ointment group $(\mathrm{p}<0.001)$ at the same time. Surgical sphincterotomy was more effective in providing early pain relief and allowing rapid healing of fissure. After 8 weeks of complete treatment $25(80.64 \%)$ patients in ointment group were asymptomatic with healed fissure in 21 $(67.74 \%)$ patients at $10^{\text {th }}$ week follow-up $(\mathrm{p}<0.05)$ in contrast to surgical sphincterotomy showing $100 \%$ pain relief as well as healing of fissure. In both groups symptomatic relief occurred earlier than fissure healing. Two female patients $(6 \%)$ in surgical group reported to have minor incontinence to flatus on stress but no incontinence was reported in ointment group $(\mathrm{p}>0.05)$. During the topical application of $0.2 \%$ glyceryl trinitrate 
a. Symptomatic relief

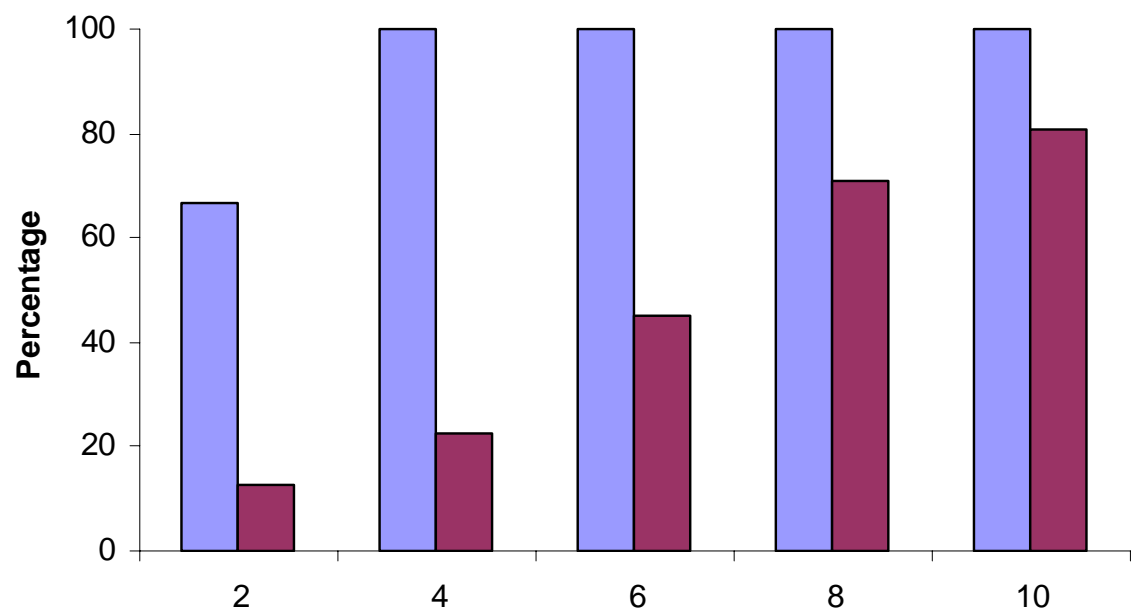

b. Healing of fissure

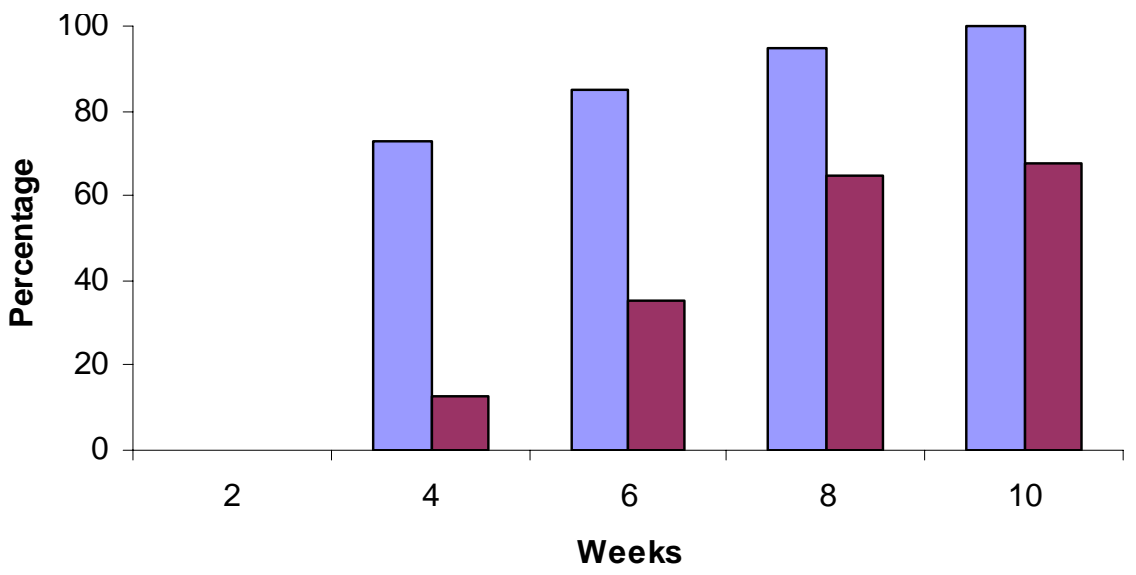

Figure 1: Comparison between symptomatic relief (a) and healing of fissure (b) following surgical sphincterotomy and local application of $0.2 \%$ glyceryl trinitrate ointment

ointment, 8 (25.80\%) patients had experienced mild headache persisting for an average of 3 days. None had reported to have incontinence to flatus or feces.

\section{Discussion}

Reduction of high resting anal tone in patients with chronic anal fissure leads to increased anodermal blood flow with resultant symptomatic relief and healing of fissure ${ }^{4}$. Lateral internal anal sphinctero- tomy is the standard surgical practice for the treatment of chronic anal fissure. However, varying degrees of anal incontinence was reported following this procedure ${ }^{3,5}$. On the other hand local application of $0.2 \%$ glyceryl trinitrate ointment can improve anodermal blood flow by inducing sphincter relaxation and thereby results in symptomatic relief and healing of fissure without compromising the anal continence. In our study, symptomatic relief occurred in $100 \%$ patients in surgical group at the end of $6^{\text {th }}$ week. Healing of 
fissure was observed in $84.85 \%$ cases during the same period rising to $100 \%$ complete healing of fissure at $10^{\text {th }}$ week. This result is fairly consistent with other studies showing a healing rate of $90 \%$ to $100 \% \%^{7,9,11}$. Carlos Parellada reported a $100 \%$ healed fissure at 10 weeks after surgical sphincterotomy ${ }^{8}$. Varying degrees of incontinence was reported to occur in $30 \%$ of patients, more frequently in women ${ }^{10}$. In our study 2 female patients $(6 \%)$ developed minor incontinence to flatus on stress. Less morbidity in our study may be procedure related where the length of incision was limited to the length of fissure and always below the dentate line.

In the present series symptomatic relief occurred at a slower pace in patients receiving topical $0.2 \%$ glyceryl trinitrate ointment. Fissure healing rate was close to a study by Lund and Scholefield (1997) with healing of fissure in two third of their patients following 8 weeks treatment with $0.2 \%$ glyceryl trinitrate ointment ${ }^{4}$. In our study one quarter of the patients experienced mild headache during the application of $0.2 \%$ glyceryl trinitrate ointment, persisting for an average of 3 days. More than half the patients were reported to have experienced headache, although these diminished in intensity with continuing application; however this side effect reduces compliance ${ }^{3}$.

Local application of $0.2 \%$ glyceryl trinitrate ointment for chronic anal fissure is safe and a high rate of healing of fissure can be achieved without the risk of incontinence, though symptomatic relief occurs at a slower rate than that of surgical sphincterotomy. It may be considered as a satisfactory option in the treatment of chronic anal fissure where there is a significant chance of incontinence especially in elderly and female patients. As surgical sphincterotomy offers better result considering early symptomatic relief and rapid healing of fissure with better patient compliance, we conclude that surgical sphincterotomy is the treatment of choice in chronic anal fissure when the procedure is performed by an experienced and skilled surgeon.

\section{Acknowledgments}

We acknowledge the contribution of our patients in the study group for their cooperation during the trial. We express our gratitude to the medical officers at the Department of Surgery, BSMMU for their continued assistance in following up the patients and data collection.

\section{References}

1. Cuschieri A, Steele RJC, Moosa AR. Essential surgical practice. $4^{\text {th }}$ ed. London, Arnold, 2002, pp 642-43.

2. Way LW, Doherty GM. Current surgical diagnosis and treatment. $12^{\text {th }}$ ed. USA, McGraw-Hill Companies, 2006, pp 750-52.

3. K.P Nugent. Benign anal disease. In: Johnson CD, Taylor I (eds). Recent advances in surgery. $25^{\text {th }}$ ed. London, Royal Society of Medicine Press Ltd, 2002, pp 147- 60 .

4. Lund JN, Scholefield JH. A randomized prospective, double-blind placebo controlled trial of glyceryl trinitrate ointment in treatment of anal fissure. Lancet 1997; 349: 11-14.

5. Lund JN, Scholcfield JH. Aetiology and treatment of anal fissure. Br J Surg. 1996; 83: 1335-44.

6. Pitt J, Williams S, Dawson PM. Reasons for failure of glyceryl trinitratc treatment of chronic fissure in ano: A multivariate analysis. Dis Colon Rectum 2001; 44: 964-67.

7. Garcia-Anguilar J, Belmonte Montes C, Perez JJ, Jensen L, Madoff RD, Wong WD. Incontinence after lateral internal sphincterotomy: Anatomic and functional evaluation. Dis Colon Rectum 1998; 41: 423-27.

8. Parellada C. Randomized, prospective trial comparing 0.2 percent isosorbide dinitrate ointment with sphincterotomy in treatment of chronic anal fissure: A two-year follow-up. Dis Colon Rectum. 2004; 47: 43743.

9. Arroyo SA, Perez VF, Miranda TE, Sanchez RA, Serrano PP, Calpena RR. Surgical (close lateral internal sphincterotomy) versus chemical (botulinum toxin) sphincterotomy as treatment of chronic anal fissure. Med Clin (Barc). 2005; 124: 573-75.

10. Nyam DC, Pemberton JH. Long-term results of lateral internal sphincterotomy for chronic anal fissure with particular reference to incidence of faecal incontinence. Dis Colon Rectum 1999; 42:1306-10.

11. Hashmat A, Ishfaq T. Chemical versus surgical sphincterotomy for chronic fissure in ano. J Coll Physi Surg Pak. 2007; 17: 44-47. 